\title{
Thermodynamic Study of Unsteady BGK Model for a Binary Gas Mixture Affected by a Nonlinear Thermal Radiation Field.
}

\section{Taha Zakaraia Abdel Wahid}

October High Institute for Engineering and Technology, $6^{\text {th }}$ October City, Giza, Egypt

Author to whom correspondence should be addressed; E-Mail: taha_zakaraia@yahoo.com; Tel.: +2-0100-8763678; Fax: +2-048-3442467

Received: 19 September 2014 / Accepted: 23 September 2014 / Published: 3 November 2014

\begin{abstract}
In the present study, a development thermodynamic study of the papers [JNET, 2011, 36 (1), 75-98 and Can. J. of Phy., 2012, 90(2): 137-149] is introduced. The non-stationary BGK (BhatnagerGross- Krook) model of the kinetic equations for a rarefied gas mixture affected by nonlinear thermal radiation field is solved instead of the stationary equations. The unsteady solution gives the problem a great generality and more applications. The non-equilibrium thermodynamic properties of the system (gas mixture + the heated plate) is investigated. The entropy, entropy flux, entropy production, thermodynamic forces, kinetic coefficients are obtained for the system. The verification of the Boltzmann H-theorem, Le Chatelier principle, second law of thermodynamic and Onsager's reciprocity relation for the system are made. The ratios between the different contributions of the internal energy changes based upon the total derivatives of the extensive parameters are estimated via the Gibbs formula. The results are applied to the Argon-Neon binary gas mixture, for various values of both of molar fraction parameters and radiation field intensity. 3D-Graphics illustrating the calculated variables are drawn to predict their behavior. The results are discussed.
\end{abstract}

Keywords: Binary gas mixture; Radiation field; Exact solutions; Travelling wave method; Unsteady BGK model; Irreversible thermodynamics.

PACS Codes: 05.70.Ln; 47.70.Mc; 51.30.+i; 47.45.Ab; 47.45.-n.

\section{Introduction}

All matter emits thermal radiation continuously, and consequently thermal radiation is an inherent part of our environment. Radiative heat transfer is important in system analysis particularly when high 
temperatures are involved, cryogenic systems are also considered, when radiation is being utilized as a source flux, or when radiative transfer is the primary mode of heat rejection. Some application examples where thermal radiation transfer is of primary importance include solar collectors, boilers and furnaces, spacecraft cooling systems, and cryogenic fuel storage systems $[1,2]$.

In spite of the fact that, the statistical-mechanical study of fluid mixtures far from equilibrium is a very interesting subject from a theoretical as well as a practical viewpoint. There are very few articles in the literature concerning this topic [3-9], compared to the huge amount of papers in the case of a single gas; see e.g. [10-16].

The particles of a gas absorb and radiate thermal energy. The interaction with the thermal radiation that is contained within the volume of the body may be important in gases, since the latter unlike solids and liquids are capable of undergoing conspicuous volume changes. Taking this interaction into account makes the behavior of the gases more realistic. Radiative heat transfer in gases has important applications from combustion systems to modeling atmospheric processes. The magnitude of radiative heat fluxes can have profound effects on combustion performance and environmental impact. For this purpose, Abourabia and Abdel Wahid [13], have introduced a new approach for studying the influence of a thermal radiation field upon a rarefied neutral gas. This idea was applied to a steady problem of the half space filled by a neutral gas specified by a flat rested heated plate. The present paper is extended to study the unsteady problem for a rarefied binary gas mixture affected by nonlinear thermal radiation field.

This new unsteady approach is made for studying the influence of thermal radiation field on a rarefied neutral binary gas mixture. Now, we have to solve two systems of partial differential equations (one for each component of the gas mixture) instead of one system of ordinary differential equations as we done before in [13].

For this purpose, we use coupled systems of unsteady kinetic BGK equations, one for each component of the binary gas mixture. The radiation field effect is inserted into the force term of the Boltzmann equation as a radiation force. These procedures are followed, using Liu-Lees model for two stream Maxwell distribution functions and the moment method. The behavior of the macroscopic properties of the binary gas mixture is predicted. This is made for various radiation field intensities due to different plate temperatures. The temperature and concentration are, in turn, substituted into the corresponding distribution functions. This approach will permit us to study the behavior of the equilibrium and non-equilibrium unsteady distribution functions for various values of the molar fraction parameters. The important non-equilibrium thermodynamic properties of the system (binary gas mixture + heated plate) are calculated. Particularly, the entropy, entropy flux, entropy production, thermodynamic forces and kinetic coefficients is obtained. The verification of the second law of thermodynamic, Boltzmann H-theorem and Onsager's reciprocity relation is made. The ratios between the different contributions of the internal energy changes are predicted via the Gibbs' formula. The results are applying to the Argon-Neon binary gas mixture. Finally the important conclusions of the paper are indicated. 


\section{The Physical Problem and Mathematical Formulation}

Consider a binary gas mixture consisting of monatomic molecules of two type's components A and B. The gas mixture fills the upper half of the space $(y \geq 0)$, which is bounded by an infinite immobile flat plate $(\mathrm{y}=0)$, in a uniform pressure $P_{S}[2,13]$. The plate is heated suddenly. This is producing thermal radiation field. The flow is considered unsteady, and compressible. In a frame co-moving with the fluid the behavior of the binary gas mixture is studied under the following assumptions:

(i) The velocities of the incident and reflected particles are equals at the rested plate boundary; but of opposite signs. This is happened according to Maxwell formula of momentum defuse reflection. On the other hand the exchange will be produced by the temperature difference between the particles and the heated plate. That is taking the form of full energy accommodation [17].

(ii) The gas is considered gray absorbing-emitting but not a scattering medium.

(iii) A thermal radiation force is acting from the heated plate on the binary atomic gas mixture, written in vector notation [17-19] as

$$
\left[\vec{F}=\frac{-4 \sigma_{s}}{3 n_{s} c} \vec{\nabla} T^{4}(y, t) \Rightarrow F_{y}=\frac{-16 \sigma_{s} T^{3}}{3 n_{s} c} \frac{\partial T(y, t)}{\partial y}\right]
$$

where $F_{y}, T, \sigma_{s}, n_{s}$ and $c$ are the thermal radiation force component along $\mathrm{y}$-axis direction, mean temperature, Stefan-Boltzmann constant, gas concentration at the plate surface and the velocity of light, respectively.

For unsteady motion, the process in the system subject to a thermal radiation force $F_{y}$ can be expressed in terms of the BGK kinetic equations in the form [10-12]:

$$
\begin{aligned}
& \frac{\partial f_{A}}{\partial t}+C_{y} \frac{\partial f_{A}}{\partial y}+\frac{F_{y}}{m_{A}} \frac{\partial f_{A}}{\partial C_{y}}=v_{A A}\left(f_{0 A}-f_{A}\right)+v_{A B}\left(f_{0 B}-f_{A}\right), \\
& \frac{\partial f_{B}}{\partial t}+C_{y} \frac{\partial f_{B}}{\partial y}+\frac{F_{y}}{m_{B}} \frac{\partial f_{B}}{\partial C_{y}}=v_{B B}\left(f_{0 B}-f_{B}\right)+v_{B A}\left(f_{0 A}-f_{B}\right),
\end{aligned}
$$

where $C_{\mathrm{y}}, f_{\beta}$ and $m_{\beta}$ are the velocity of the gas particles component along $\mathrm{y}$-axis, the two-stream Maxwell distribution function and mass of particles of the $\beta$ type. The four quantities $v_{A A}, v_{A B}, v_{B B}$ and $v_{B A}$ are the collision frequencies that are given in [12,20-22] as:

$$
\begin{aligned}
& v_{\alpha \alpha}=n_{s \alpha} \sigma_{\alpha \alpha} V_{p \alpha} \quad \text { and } \quad v_{\alpha \beta}=n_{s \alpha} \sigma_{\alpha \beta} V_{p \alpha \beta} \text { where } \quad V_{p \alpha}=\left[\sqrt{\frac{8 K T_{s}}{\pi m_{\alpha}}}\right], \quad V_{p \alpha \beta}=\left[\sqrt{\frac{8 K T_{s}}{\pi \mu_{\alpha \beta}}}\right], \\
& \mu_{A B}=\left(\frac{m_{A} m_{B}}{m_{A}+m_{B}}\right), \sigma_{A B}=\pi d_{e f f}^{2}, d_{e f f}=\left(\frac{d_{A}+d_{B}}{2}\right)^{2},(\alpha \text { and } \beta \equiv A \text { or } B),
\end{aligned}
$$

where $V_{p \alpha}, \mu_{\alpha \beta}, d_{e f f}, n_{s \alpha}$ and $\sigma_{\alpha \beta}$ are the mean velocity of particles, the reduced mass, the diameter of the effective collisions sphere, gas concentration at the plate surface, and effective collisions cross section for $\alpha$ and $\beta$ types, respectively. 
The local Maxwell distribution functions $f_{0 \beta}$ are denoted by:

$f_{0 \beta}=\frac{n_{\beta}}{\left(2 \pi R T_{\beta}\right)^{\frac{3}{2}}} \exp \left[\frac{-C^{2}}{\left(2 R T_{\beta}\right)}\right]$, where $R$ is the gas constant and $C^{2}=C_{x}^{2}+C_{y}{ }^{2}+C_{z}^{2}$.

Lee's moment method [23-24] is employed here to obtain the solution of the BGK equation. When the application of heat to a gas. It to expand, it is thereby rendered rarer than the neighboring parts of the gas; and it tends to form an upward current of the heated gas, which is of course accompanied with a current of the more remote parts of the gas in the opposite direction. The fresh portions of gas are brought into the neighborhood of the source of heat, carrying their heat with them into other regions [25]. We assume that the temperature of the upward going gas particles is $T_{1 \beta}$, while the temperature of the downward going gas particles is $T_{2 \beta}[\mathbf{1 3}, \mathbf{2 6}]$. The corresponding concentrations are $n_{1 \beta}$ and $n_{2 \beta}$. We use the Liu-Lees model of the two-stream Maxwell distribution function near the plate [17,27] for particles of the $\beta$ type, which can be represented as:

$f_{\beta}=\left\{\begin{array}{l}f_{1 \beta}=\frac{n_{1 \beta}}{\left(2 \pi R T_{1 \beta}\right)^{\frac{3}{2}}} \exp \left[\frac{-C^{2}}{\left(2 R T_{1 \beta}\right)}\right], \text { For } C_{y}>0 \uparrow \\ f_{2 \beta}=\frac{n_{2 \beta}}{\left(2 \pi R T_{2 \beta}\right)^{\frac{3}{2}}} \exp \left[\frac{-C^{2}}{\left(2 R T_{2 \beta}\right)}\right], \text { For } C_{y}<0 \quad \downarrow\end{array}\right.$.

We are interesting with the moments of the distribution function. Therefore, we derive the Maxwell's moment equations by multiplying the BGK equation by a function of velocity $Q_{i}(C)$ and integrating over velocity space. How many and what forms of $Q_{i}$ will be used is dependent on how many unknown variables need to be determined and how many equations need to be solved. Multiplying Eqs. $(2,3)$ by some functions of velocity $Q_{i}=Q_{i}(C)$, and integrating with respect to $C$. The discontinuity of the distribution function, caused by the cone of influence [17], is taken into consideration. The resulting equation can then be written as follows: for particles of each gas component $\beta$ with the second one $\alpha$ type,

$$
\begin{aligned}
& \frac{\partial}{\partial t}\left[\int_{-\infty}^{\infty} \int_{-\infty}^{0} \int_{-\infty}^{\infty} Q_{i} f_{2 \beta} d \underline{c}+\int_{-\infty}^{\infty} \int_{0}^{\infty} \int_{-\infty}^{\infty} Q_{i} f_{1 \beta} d \underline{c}\right]+\frac{d}{d y}\left[\int_{-\infty}^{\infty} \int_{-\infty}^{0} \int_{-\infty}^{\infty} Q_{i} C_{y} f_{2 \beta} d \underline{c}+\int_{-\infty}^{\infty} \int_{0}^{\infty} \int_{-\infty}^{\infty} Q_{i} C_{y} f_{1 \beta} d \underline{c}\right] \\
& -\frac{F_{y}}{m_{\beta}}\left[\int_{-\infty}^{\infty} \int_{-\infty}^{0} \int_{-\infty}^{\infty} f_{2 \beta} \frac{d Q_{i}}{d C_{y}} d \underline{c}+\int_{-\infty}^{\infty} \int_{0}^{\infty} \int_{-\infty}^{\infty} f_{1 \beta} \frac{d Q_{i}}{d C_{y}} d \underline{c}\right]=v_{\beta \beta}\left[\int_{-\infty}^{\infty} \int_{-\infty}^{\infty} \int_{-\infty}^{\infty} Q_{i} f_{0 \beta} d \underline{c}-\right. \\
& \left.\left(\int_{-\infty}^{\infty} \int_{-\infty}^{0} \int_{-\infty}^{\infty} f_{2 \beta}+\int_{-\infty}^{\infty} \int_{0}^{\infty} \int_{-\infty}^{\infty} f_{1 \beta}\right) Q_{i} d \underline{c}\right]+v_{\beta \alpha}\left[\int_{-\infty}^{\infty} \int_{-\infty}^{\infty} \int_{-\infty}^{\infty} Q_{i} f_{0 \alpha} d \underline{c}-\left(\int_{-\infty}^{\infty} \int_{-\infty}^{0} \int_{-\infty}^{\infty} f_{2 \beta}+\int_{-\infty}^{\infty} \int_{0}^{\infty} \int_{-\infty}^{\infty} f_{1 \beta}\right) Q_{i} d \underline{c}\right],
\end{aligned}
$$

where $d \underline{c}=d C_{x} d C_{y} d C_{z}$.

Equations (5) are called the general equations of transfer [28]. Using the dimensionless forms by taking: 


$$
\begin{aligned}
& y=\bar{y}\left(\tau_{A B} \sqrt{2 R T_{s}}\right), t=\bar{t} \tau_{A B}, \vec{C}=\overline{\vec{C}} \sqrt{2 R T_{s}}, f_{i \beta}=\frac{\overline{f_{i \beta}}\left(2 \pi R T_{s}\right)^{\frac{3}{2}}}{n_{s}}, i=0,1,2 \text {, } \\
& N_{\beta}=\frac{16 \sigma_{s} T_{s}^{3}}{3 n_{s} c m_{\beta} R}, T_{1 \beta}=\bar{T}_{1 \beta} T_{s}, n_{1 \beta}=\bar{n}_{1 \beta} n_{s}, T_{2 \beta}=\bar{T}_{2 \beta} T_{s}, \quad n_{2 \beta}=\bar{n}_{2 \beta} n_{s} \text {, } \\
& \text { and } d U_{\beta}=\overline{d U_{\beta}} K_{b} T_{s} \text {. }
\end{aligned}
$$

where $\tau_{A B}, N_{\beta}$ and $d U_{\beta}$ are the relaxation time between collisions of the A-B species, nondimensional constant and internal energy change of the gas species of the $\beta$ type, respectively.

Now, we can take $Q_{i}=C^{2}$ and $\frac{1}{2} C_{y} C^{2}$. Substituting formula (4) into the transfer Eq.(5), using (6), we get the moments of the BGK equations in dimensionless form.

After dropping the bars we have eight equations, four for each component of the binary gas mixture as follows: For particles of the $\beta=A, B$ types, the conservation of energy is

$$
\begin{aligned}
& \frac{\partial}{\partial t}\left(n_{1 \beta} T_{1 \beta}+n_{2 \beta} T_{2 \beta}\right)+\frac{\partial}{\partial y}\left[\left(n_{1 \beta} T_{1 \beta}^{\frac{3}{2}}-n_{2 \beta} T_{2 \beta}{ }^{\frac{3}{2}}\right)\right]+N_{\beta}\left(n_{1 \beta} T_{1 \beta}^{\frac{1}{2}}-n_{2 \beta} T_{2 \beta}{ }^{\frac{1}{2}}\right) \\
& \left(\frac{n_{1 \beta} T_{1 \beta}+n_{2 \beta} T_{2 \beta}}{n_{1 \beta}+n_{2 \beta}}\right)^{3} \frac{\partial}{\partial y}\left(\frac{n_{1 \beta} T_{1 \beta}+n_{2 \beta} T_{2 \beta}}{n_{1 \beta}+n_{2 \beta}}\right)=0,
\end{aligned}
$$

The heat flux component in the y-direction is

$$
\begin{aligned}
& \frac{\partial}{\partial t}\left(n_{1 \beta} T_{1 \beta}^{\frac{3}{2}}-n_{2 \beta} T_{2 \beta}{ }^{\frac{3}{2}}\right)+\frac{5}{4} \frac{\partial}{\partial y}\left[\left(n_{1 \beta} T_{1 \beta}^{2}+n_{2 \beta} T_{2 \beta}^{2}\right)\right]+ \\
& \frac{3}{2} N_{\beta}\left(n_{1 \beta} T_{1 \beta}+n_{2 \beta} T_{2 \beta}\right)\left(\frac{n_{1 \beta} T_{1 \beta}+n_{2 \beta} T_{2 \beta}}{n_{1 \beta}+n_{2 \beta}}\right)^{3} \frac{\partial}{\partial y}\left(\frac{n_{1 \beta} T_{1 \beta}+n_{2 \beta} T_{2 \beta}}{n_{1 \beta}+n_{2 \beta}}\right)= \\
& {\left[\frac{v_{\beta \beta}}{v_{A B}}\left(n_{1 \beta} T_{1 \beta}^{\frac{3}{2}}-n_{2 \beta} T_{2 \beta}^{\frac{3}{2}}\right)+\frac{v_{\beta \alpha}}{v_{A B}}\left(n_{1 \beta} T_{1 \beta}^{\frac{3}{2}}-n_{2 \beta} T_{2 \beta}^{\frac{3}{2}}\right)\right] .}
\end{aligned}
$$

The above two equations, for both groups of equations, corresponding to each species, are complemented by the equations of state:

$$
P_{\beta}=n_{\beta} T_{\beta}=\text { const., }
$$

We shall study the problem in a coordinate system of the phase space in which the bulk velocity $u$ is located at the origin. Thus, we get the fourth equation:

$$
\left(n_{1 \beta} T_{1 \beta}^{\frac{1}{2}}-n_{2 \beta} T_{2 \beta}^{\frac{1}{2}}\right)=0
$$


Travelling wave solution method [29-30] is used to solve the problem, considering $\xi=l y-m t$

Such that to make all the dependent variables as functions of $\xi$. Here $l$ and $m$ are transformation constants, which do not depend on the properties of the fluid but as parameters to be determined by the boundary and initial conditions [29-30]. Using Eq.(11) we get the derivatives:

$\frac{\partial}{\partial t}=-m \frac{\partial}{\partial \xi}, \frac{\partial}{\partial y}=l \frac{\partial}{\partial \xi}$ and $\frac{\partial^{a}}{\partial t^{a}}=(-1)^{a} m^{a} \frac{\partial^{a}}{\partial \xi^{a}}, \frac{\partial^{a}}{\partial y^{a}}=l^{a} \frac{\partial^{a}}{\partial \xi^{a}}$,

where $a$ is positive integer. Substituting from Eqs. (11-12), into Eqs. (7-8) we get:

$$
\begin{aligned}
& -m \frac{\partial}{\partial \xi}\left(n_{1 \beta} T_{1 \beta}+n_{2 \beta} T_{2 \beta}\right)+l \frac{\partial}{\partial \xi}\left[\left(n_{1 \beta} T_{1 \beta}^{\frac{3}{2}}-n_{2 \beta} T_{2 \beta}^{\frac{3}{2}}\right)\right] \\
& +l N_{\beta}\left(\left(\frac{n_{1 \beta} T_{1 \beta}+n_{2 \beta} T_{2 \beta}}{n_{1 \beta}+n_{2 \beta}}\right) \frac{\partial}{\partial \xi}\left(\frac{n_{1 \beta} T_{1 \beta}+n_{2 \beta} T_{2 \beta}}{n_{1 \beta}+n_{2 \beta}}\right)\right)\left(n_{1 \beta} T_{1 \beta}^{\frac{1}{2}}-n_{2 \beta} T_{2 \beta}^{\frac{1}{2}}\right)=0 \\
& -m \frac{\partial}{\partial \xi}\left(n_{1 \beta} T_{1 \beta}^{\frac{3}{2}}-n_{2 \beta} T_{2 \beta}^{\frac{3}{2}}\right)+\frac{5}{4} l \frac{\partial}{\partial \xi}\left[\left(n_{1 \beta} T_{1 \beta}^{2}+n_{2 \beta} T_{2 \beta}^{2}\right)\right] \\
& +\frac{3}{2} l N_{\beta}\left(\left(\frac{n_{1 \beta} T_{1 \beta}+n_{2 \beta} T_{2 \beta}}{n_{1 \beta}+n_{2 \beta}}\right)^{3} \frac{\partial}{\partial \xi}\left(\frac{n_{1 \beta} T_{1 \beta}+n_{2 \beta} T_{2 \beta}}{n_{1 \beta}+n_{2 \beta}}\right)\right)\left(n_{1 \beta} T_{1 \beta}+n_{2 \beta} T_{2 \beta}\right)= \\
& {\left[\frac{v_{\beta \beta}}{v_{A B}}\left(n_{1 \beta} T_{1 \beta}^{\frac{3}{2}}-n_{2 \beta} T_{2 \beta}^{\frac{3}{2}}\right)+\frac{v_{\beta \alpha}}{v_{A B}}\left(n_{1 \beta} T_{1 \beta}^{\frac{3}{2}}-n_{2 \beta} T_{2 \beta}^{\frac{3}{2}}\right)\right],}
\end{aligned}
$$

Eqs. $(9,10,13,14)$ are solved to obtain the solution of the initial and boundary value problem for each gas components to estimate the four unknowns $T_{1 \beta}, T_{2 \beta}, n_{1 \beta}$ and $n_{2 \beta}$.

The values of the integrated constants can be estimated under the initial and boundary conditions (as $(y, t)=(0,0) \Rightarrow \xi=0)$ :

$\frac{\left(n_{1 \beta}(\xi=0)+n_{2 \beta}(\xi=0)\right)}{2}=C_{\beta}$, where $C_{\beta}=\frac{n_{s \beta}}{n_{s}}$ is the molar fraction.

$\left(\frac{n_{1 \beta}(\xi=0) T_{1 \beta}(\xi=0)+n_{2 \beta}(\xi=0) T_{2 \beta}(\xi=0)}{n_{1 \beta}(\xi=0)+n_{2 \beta}(\xi=0)}\right)=1$,

$$
\left(n_{1 \beta}(\xi=0) T_{1 \beta}(\xi=0)^{\frac{1}{2}}-n_{2 \beta}(\xi=0) T_{2 \beta}(\xi=0)^{\frac{1}{2}}\right)=0
$$


The temperature of the incident particles is assumed to be $T_{2}$ while the temperature of the reflected particles from the plate is the temperature $T_{1}$, they are related such that [31-32]:

$T_{2 \beta}(\xi=0)=\chi_{\beta} T_{1 \beta}(\xi=0): 0<\chi_{\beta} \leq 1$,

where $\chi_{\beta}$ is the ratio between the plate and gas temperatures. The parameter $\chi_{\beta}$ can take arbitrary positive value less than unity as the plate is hotter than the gas. We can obtain by solving the algebraic system of Eqs. (15-18) that

$$
\begin{aligned}
& n_{1 \beta}(\xi=0)=\left(2 C_{\beta}-\frac{2 C_{\beta}}{1+\sqrt{\chi_{\beta}}}\right), n_{2 \beta}(\xi=0)=\left(\frac{2 C_{\beta}}{1+\sqrt{\chi_{\beta}}}\right), \\
& T_{1 \beta}(\xi=0)=\left(\frac{1}{\sqrt{\chi_{\beta}}}\right) \text { and } T_{2 \beta}(\xi=0)=\left(\sqrt{\chi_{\beta}}\right) .
\end{aligned}
$$

The above four quantities represent the initial and boundary conditions.

Introducing the obtained quantities $T_{1}, T_{2}, n_{1}$ and $n_{2}$ into the two stream Maxwell distribution function where;

$$
\left\{\begin{array}{l}
f_{1 \beta}=\frac{n_{1 \beta}}{T_{1 \beta}{ }^{\frac{3}{2}}} \exp \left[\frac{-C^{2}}{T_{1 \beta}}\right], \text { For } C_{y}>0 \\
f_{2 \beta}=\frac{n_{2 \beta}}{T_{2 \beta}{ }^{\frac{3}{2}}} \exp \left[\frac{-C^{2}}{T_{2 \beta}}\right], \text { For } C_{y}<0
\end{array},\right.
$$

we can get the sought distribution functions. These estimated distribution functions of the gas particles enable us to study their behavior in the considered system, which is not possible by taking the way of the solution of Navier-Stokes equations. This will be the starting point to predict the irreversible thermodynamic behavior of the system in the next section.

\section{The Non-Equilibrium Thermodynamic Properties of The System:}

The everyday resorts to the linear theory of the thermodynamics of irreversible processes still constitute great interests [33-35]. This is associated both with the general theoretical importance of this theory and its numerous applications in various branches of science. It is unquestionable that the concept of entropy has played an essential role both in the physical and biological sciences [36]. Thus, we start the thermodynamic investigations of the problem from the evaluation of the entropy $S$ per unit mass of the binary gas, which is written as [37]: 
$S(y, t)=\rho^{-1} \sum_{\beta=A}^{B} \rho_{\beta} S_{\beta}$, where $S_{\beta}$ is the entropy of the gas species of the $\beta$ type denoted by [38], $S_{\beta}(y, t)=-\int f_{\beta} \log f_{\beta} d \underline{c}$. The y-component of the entropy flux vector has the form [39], $J_{y}(y, t)=\sum_{\beta=A}^{B} J_{y \beta}(y, t)$ where $J_{y \beta}$ is the y-component of the entropy flux of the gas species of the $\beta$ type denoted by, $J_{y \beta}(y, t)=-\int c_{y} f_{\beta} \log f_{\beta} \underline{d c}$, while the Boltzmann's entropy production [33-35] in the unsteady state $\sigma_{S}$ is expressed as,

$\sigma_{S}(y, t)=\frac{\partial S(y, t)}{\partial t}+\underline{\nabla} \cdot \underline{J}(y, t)$. Following the general theory of thermodynamic [40-45], we could estimate the thermodynamic forces; the first corresponding to the change in the concentration $X_{n}$, as: $X_{n}(y, t)=\frac{\Delta y}{n(y, t)} \frac{\partial n(y, t)}{\partial y}$, the second thermodynamic force corresponding to the change in the temperature $X_{T}$, as $[2,7,13]$ :

$X_{T}(y, t)=\frac{\Delta y}{T(y, t)} \frac{\partial T(y, t)}{\partial y}$ and the third thermodynamic force corresponds to the change in the radiation field energy $X_{R}$, as $[2,7,13], X_{R}(y, t)=\frac{\Delta y}{U_{R}(y, t)} \frac{\partial U_{R}(y, t)}{\partial y}$,

where $U_{R \beta}(y, t)=\left(\frac{16 \sigma T_{s}^{4}}{3 c n_{s} K_{B} T_{s}}\right) T_{\beta}^{4}(y, t)$ is the dimensionless radiation field energy influences the gas particles and $\Delta y$ is the thickness of the layer adjacent to the flat plate in units of the mean free path-the distance between two collisions of the gas particles-in dimensionless form.

After calculating the thermodynamic forces and the entropy production, we can obtain the kinetic coefficients $L_{i j}$ from the relationship between the entropy production and the thermodynamic forces, via the form $[34,35,46]$ :

$\sigma_{S}(y, t)=\sum_{i} \sum_{j} L_{i j} X_{i} X_{j}=\left(\begin{array}{lll}X_{1} & X_{2} & X_{3}\end{array}\right)\left(\begin{array}{lll}L_{11} & L_{12} & L_{13} \\ L_{21} & L_{22} & L_{23} \\ L_{31} & L_{32} & L_{33}\end{array}\right)\left(\begin{array}{l}X_{1} \\ X_{2} \\ X_{3}\end{array}\right) \geq 0$.

This constitute the restriction on the sign of phenomenological coefficients $L_{i j}$, which arise as a consequence of the second law of the thermodynamics [34], which can be deduced from the standard results in algebra. The necessary and sufficient conditions for $\sigma_{s}(y, t) \geq 0$ are fulfilled by the determinant, $\left|L_{i j}+L_{j i}\right| \geq 0$, and all its principal minors are non-negative too. Another restriction on $L_{i j}$ was established by Onsager (1931). He found that, besides the restriction on the sign, the phenomenological coefficients verify important symmetry properties. Invoking the principle of microscopic reversibility and using the theory of fluctuations, Onsager was able to demonstrate the symmetry property denoted by, $L_{i j}=L_{j i}$, which is called the Onsager's reciprocal relations.

The Gibbs formula for the variation of the internal energy applied to the system (binary gas mixture + heated plate), $d U(y, t)$ is

$d U(y, t)=d U_{S}(y, t)+d U_{V}(y, t)+d U_{R}(y, t)$. 
The internal energy change due to the variation of the extensive variables, such as entropy $d U_{S}$, volume $d U_{V}$ and in addition the temperature gradient produced by the thermal radiation field $d U_{R}$, are respectively read for a binary gas [37], as follows:

$d U_{S}(y, t)=\rho^{-1} \sum_{\beta=A}^{B} \rho_{\beta} d U_{S \beta}$, where $d U_{S \beta}(y, t)=T_{\beta} d S_{\beta}$,

$d U_{V}(y, t)=\rho^{-1} \sum_{\beta=A}^{B} \rho_{\beta} d U_{V \beta}$, where $d U_{V \beta}(y, t)=-P_{\beta} d V_{\beta}$,

$d U_{R}(y, t)=\rho^{-1} \sum_{\beta=A}^{B} \rho_{\beta} d U_{R \beta}$, where $d U_{R \beta}(y, t)=\omega_{\beta} \frac{\partial T_{\beta}^{4}(y, t)}{\partial y} \Delta y, \omega_{\beta}=\frac{16 \sigma T_{s}{ }^{4}}{3 c n_{s} K_{B} T_{s}}$.

The pressure and change in volume are $P_{\beta}=n_{\beta} T_{\beta}$ and $d V_{\beta}=-\frac{d n_{\beta}}{n_{\beta}{ }^{2}}$ respectively, and $d n=\frac{\partial n}{\partial t} \delta t+\frac{\partial n}{\partial y} \delta y, d S=\frac{\partial S}{\partial t} \delta t+\frac{\partial S}{\partial y} \delta y$ and $\delta y=1, \delta t=1$

\section{Results and Discussion}

We investigated the behavior of the binary gas mixture under the influence of a non-linear thermal radiation field in the unsteady state of a plane heat transfer problem in the

system (binary gas mixture + heated plate). The thermal radiation is introduced, in the force term of the Boltzmann equation for a rarefied gas subject to the inequalities [47], $\lambda \square \delta \square d$, where $\delta=n^{-1 / 3}$ is the average spacing between the molecules, both $\lambda$ and $d$ are the mean free path and the molecular diameter, respectively. In all calculations and figures, we take the following parameters values from [48, 49] for the Argon-Neon binary gas mixture, where the particles of the A and B components are the argon and neon gases, respectively:

$T_{s}=1000 K ; \sigma_{s}=5.6705 \times 10^{-5} \mathrm{~J} \cdot \mathrm{m}^{-2} \cdot \mathrm{sec}^{-1} \cdot \mathrm{K}^{-4} ; R=8.3145 \mathrm{~J} \cdot \mathrm{K}^{-1} \cdot \mathrm{mol}^{-1}$;

$K_{B}=1.3807 \times 10^{-23} \mathrm{~J} . \mathrm{K}^{-1} ; c=2.9979 \times 10^{8} \mathrm{~m} \cdot \mathrm{sec}^{-1} ; n_{s}=3 \times 10^{18} \mathrm{~m}^{-3} ;$

$m_{A}=39.948 m_{u} ; m_{B}=20.183 m_{u} ; m_{u}=1.6605 \times 10^{-27} \mathrm{Kg}$ is atomic mass unit;

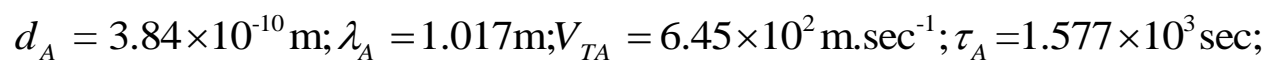

$d_{B}=2.425 \times 10^{-10} \mathrm{~m} ; \lambda_{B}=2.551 \mathrm{~m} ; V_{T B}=9.077 \times 10^{2} \mathrm{~m} \cdot \mathrm{sec}^{-1} ; \tau_{B}=2.81 \times 10^{3} \mathrm{sec} ;$

for a fixed $C_{A}=0.5 ; N_{A}(1000 \mathrm{~K})=0.609694, N_{B}(1000 \mathrm{~K})=1.20676$,

where $K_{B}, V_{T \beta}$ and $\tau_{\beta}$ are Boltzmann constant, thermal velocity, and the relaxation time of the gas species of the $\beta$ type, respectively.

Although we calculate all the sought variables in three various radiation field intensities due to different plate temperatures ( $T=800 \mathrm{~K}, 1000 \mathrm{~K}, 1400 \mathrm{~K})$, we particularize our graphics in one case corresponding to $(T=1000 \mathrm{~K})$, to economize the figures and the illustrations.

All figures show that all variables satisfy the equilibrium condition where in the equilibrium as $\chi=1$ :

$n(\chi=1)=1, T(\chi=1)=1, S(\chi=1)=I t$ 's maxmum value, $\sigma(\chi=1)=0, d U_{S}(\chi=1)=0$, $d U_{V}(\chi=1)=0, d U_{R}(\chi=1)=0, X_{T}(\chi=1)=0, X_{n}(\chi=1)=0, X_{R}(\chi=1)=0$,

see figures

(1 to 8 -a).

Now, we will discuss the behavior of the gas particles far from the equilibrium state; while the number density $n$ decreases, with time, the temperature $T$ increases, these happen for all values of $\chi$. This is 
due to the fact of the uniform pressure, see figures (1,2-a). Similarly, while the number density $n$ increases, with time, the temperature $T$ decreases, these happen for all values of $C_{A}$. This is due to the fact of the uniform pressure, see figures $(1,2-b)$. The entropy $S$ always increases with time and the entropy production $\sigma$ has a nonnegative values for all values of $t, \chi$ and the molar fraction $C_{A}$. This gives a complete satisfaction of the second law of thermodynamics and the Boltzmann H-theorem. This behavior agrees with the famous Le-Chatelier principle.

The behavior of the different contributions of the internal energy change can be illustrated in figures (3, $4,5-a, b)$. The numerical ratios between the different contributions of the internal energy changes based upon the total derivatives of the extensive parameters are predicted via the Gibbs formula. Taking into consideration their different tendencies, the maximum numerical values of the three contributions at various radiation field intensity (corresponding to various plate temperatures), are ordered in magnitude as follows:

(a) For a fixed value $C_{A}=0.5$ and variable values of $\chi$ in the considered range $(0.65<\chi<1)$, we have $d U_{s}(1000 \mathrm{~K}): d U_{V}(1000 \mathrm{~K}): d U_{R}(1000 \mathrm{~K}) \square 1: 0.333: 3.333$, see figures (3-a, 4-a, 5-a).

Figure (3-a) indicated that, $d U_{R}$ reaches its maximum numerical value at $\chi=0.65$ which means that the more the temperature difference (between the plate surface temperature and the binary gas mixture temperature) the more will be the effective contribution of the thermal radiation energy in the system total energy change. This is attributed to the behavior of the corresponding values of the thermodynamic force $X_{R}$ itself, see figure (8-a).

(b) For a fixed value $(\chi=2 / 3)$ and variable values of $C_{A}$ in the considered range $\left(0.2<C_{A}<0.95\right)$, we have $d U_{s}(1000 \mathrm{~K}): d U_{V}(1000 \mathrm{~K}): d U_{R}(1000 \mathrm{~K}) \square 1: 0.132: 1.32$,

see figures (3-b, 4-b, 5-b). The contribution of $d U_{R}$ reaches its maximum numerical value at the values of $C_{A}=0.2$ and $C_{B}=0.8$ which represent indirectly the concentrations of the heavier gas (Argon), and the lighter gas (Neon) respectively. This means that, the more the lighter mass of the gas species, the more the effectiveness of the thermal radiation energy contribution in the system total energy change, see figure (3-b). This is also due to the behavior of the corresponding value of the thermodynamic force $X_{R}$ itself corresponding to each component in the binary gas mixture, see figure (8-b). The thermodynamic force $X_{T}$ (due to the gradient of temperature) will have the opposite direction to the thermodynamic force $X_{n}$ (due to the gradient of the density), see figures $(6,7-\mathrm{a}, \mathrm{b})$. This gives a qualitative agreement in the behavior with the thermodynamic forces determined in [48], comparing with the same gas mixture (Ar-Ne) at the same molar fraction $C_{A}=0.5$.

According to our calculations, the restrictions imposed on the kinetic coefficients $L_{i j}$ are satisfied where $L_{11} \geq 0, L_{22} \geq 0$ and $L_{33} \geq 0$, for all values in the considered ranges of both $\chi$ and $C_{A}$. Onsager's reciprocal relations are satisfied, as we have $\left(L_{12} \equiv L_{21}, L_{13} \equiv L_{31}\right.$ and $\left.L_{32} \equiv L_{23}\right)$, for all values in the considered ranges of both $\chi$ and $C_{A}$.

\section{Conclusions}

By analyzing the obtained results, we conclude that:

a) The key controlling factors, which are, directly, proportional to the effectiveness of the radiation energy in the system total energy change, are: 
i) The ratio between temperature of the plate surface and the gas mixture particles, ii) The mass ratio between each component of the binary gas mixture, iii) The molar fraction of each component of the mixture.

b) At a relatively high temperature ( $T \geq 1000 \mathrm{~K})$, the radiation energy contribution in the total internal energy change becomes the dominant one and cannot be ignored at all.

c) At a relatively small temperature $(T<600 \mathrm{~K})$, the radiation energy contribution in the total internal energy change become less by orders of magnitude than the other kinds of energy contributions.

d) The lighter gas component (Neon) is affected by the non-linear thermal radiation field more than for the heavier one (Argon).

e) The second law of thermodynamics, the Boltzmann H-theorem, the Le-Chatelier principle and the Onsager's reciprocal relations, are all satisfied for the studied binary mixture system.

f) The negative sign at some kinetic coefficients, corresponding to cross effects, imply in such cases that there is a heat flux opposite to the main flux due to the imposed thermodynamic force (gradient). For example, the negative sign in front of $L_{T n}=L_{12}$ and $L_{R n}=L_{13}$, implies that there is a flow caused by the temperature gradient, from a lower to a higher temperature, known as thermal diffusion (or Soret effect) which gives a qualitative agreement with the results of study [48].

Figure 1. (a) Concentration $n$ vs. $t$ and $\chi$ at $C_{A}=0.5, \mathrm{y}=0.3$. (b) Concentration $n$ vs. $t$ and $C_{A}$ at $\chi=0.66, \mathrm{y}=0.3$.
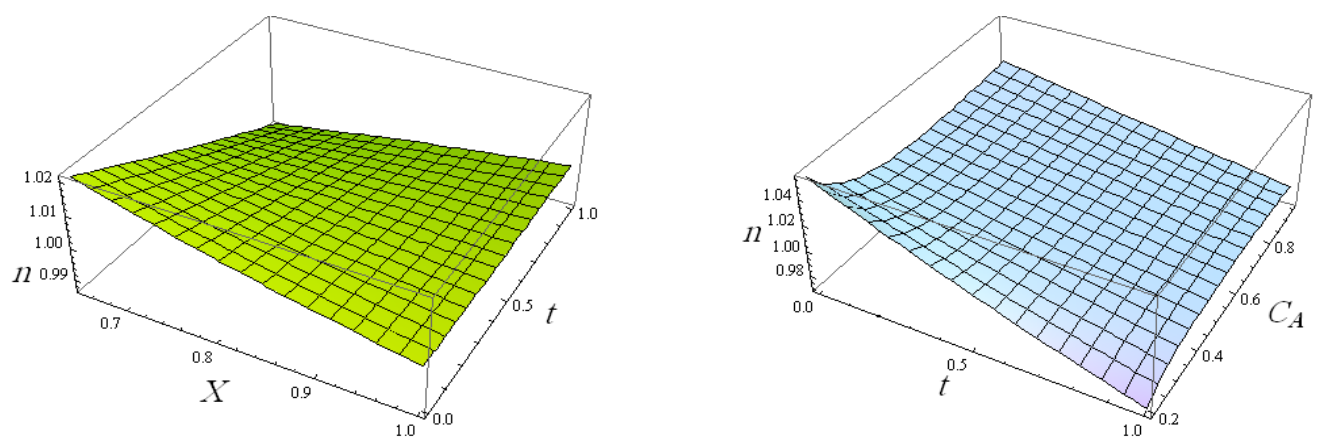

Figure 2. (a) Temperature $T$ vs. $t$ and $\chi$ at $C_{A}=0.5, \mathrm{y}=0.3$. (b) Temperature $T$ vs. $t$ and $C_{A}$ at $\chi=0.66, \mathrm{y}=0.3$.
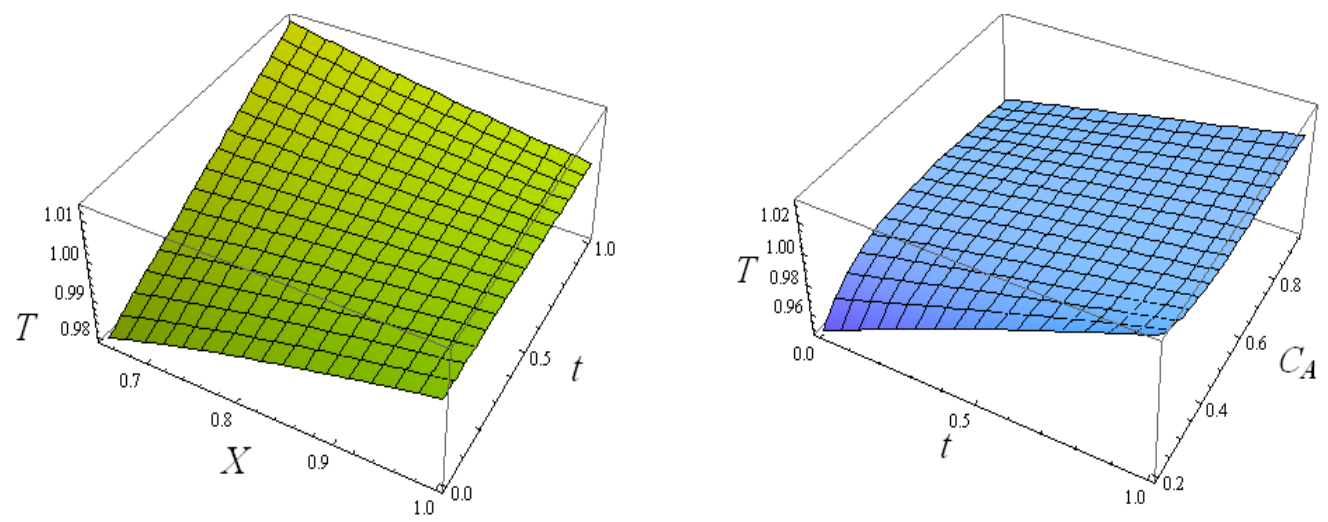
Figure 3. (a): $d U_{S}$ vs. $t$ and $\chi$ at $C_{A}=0.5, \mathrm{y}=0.3$. (b) $d U_{S}$ vs. $t$ and $C_{A}$ at $\chi=0.66, \mathrm{y}=0.3$.
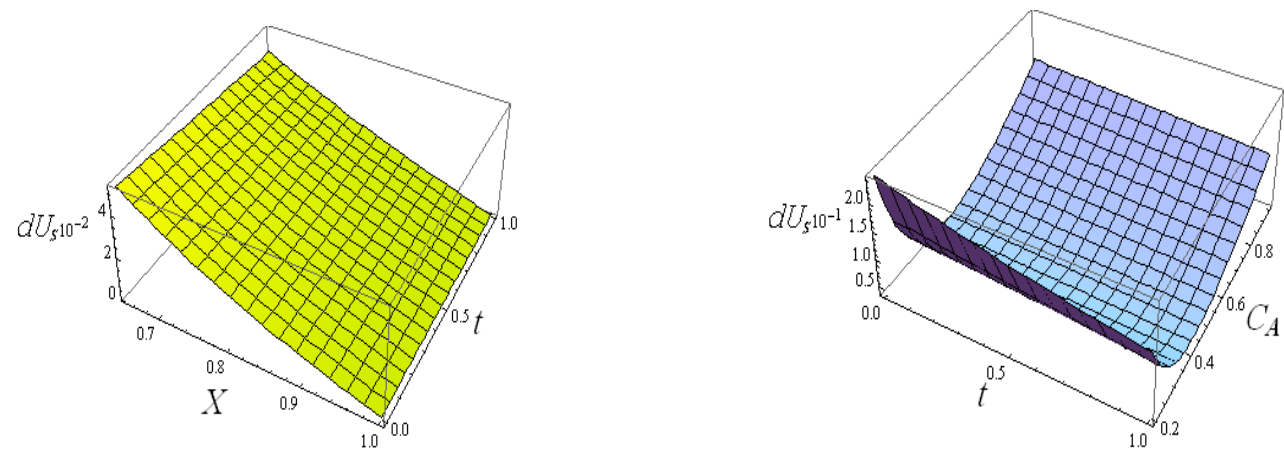

Figure 4. (a) $d U_{v}$ vs. $t$ and $\chi$ at $C_{A}=0.5, \mathrm{y}=0.3$. (b) $d U_{v}$ vs. $t$ and $C_{A}$ at $\chi=0.66, \mathrm{y}=0.3$.
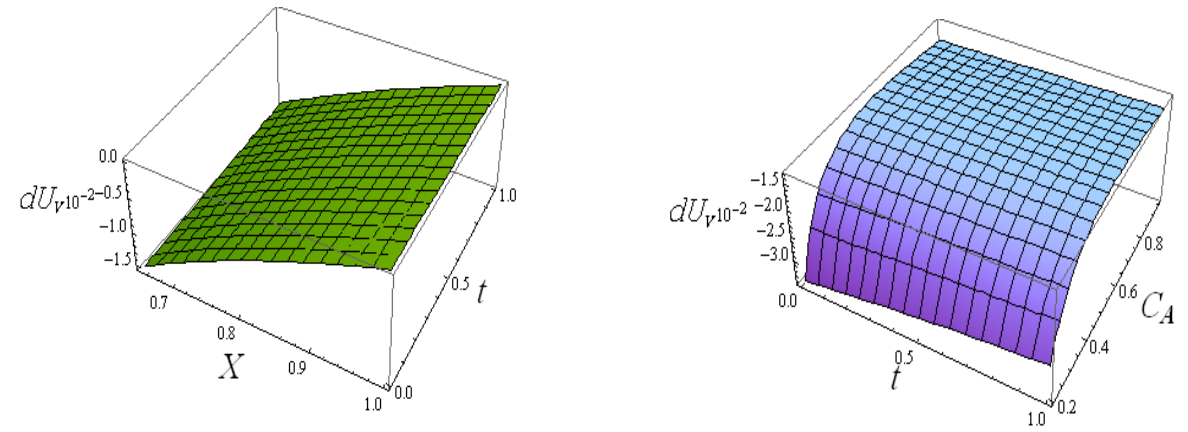

Figure 5. (a): $d U_{R}$ vs. $t$ and $\chi$ at $C_{A}=0.5, \mathrm{y}=0.3$. (b) $d U_{R}$ vs. $t$ and $C_{A}$ at $\chi=0.66, \mathrm{y}=0.3$.
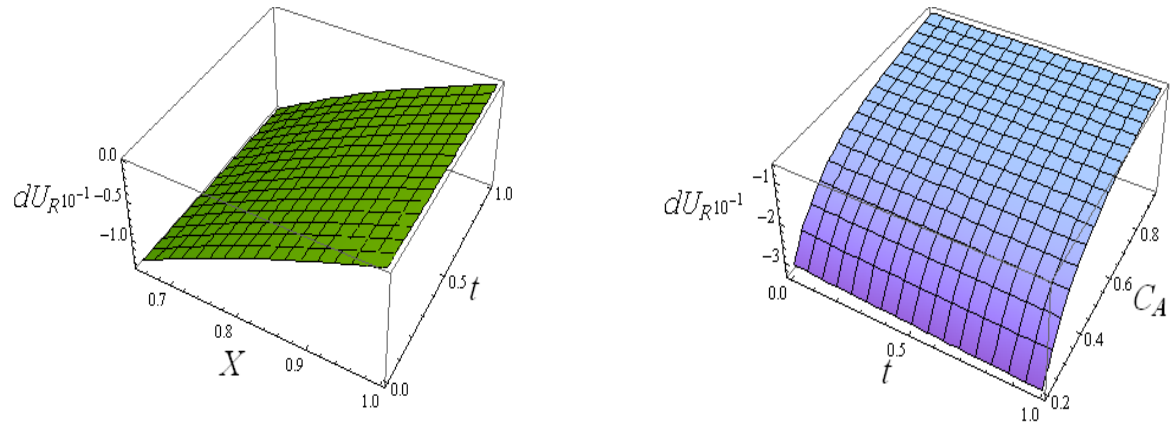
Figure 6. (a) Thermodynamic force $X_{T}$ vs. $t$ and $\chi$ at $C_{A}=0.5, \mathrm{y}=0.3$. (b) Thermodynamic force $X_{T}$ vs. $t$ and $C_{A}$ at $\chi=0.66, \mathrm{y}=0.3$.
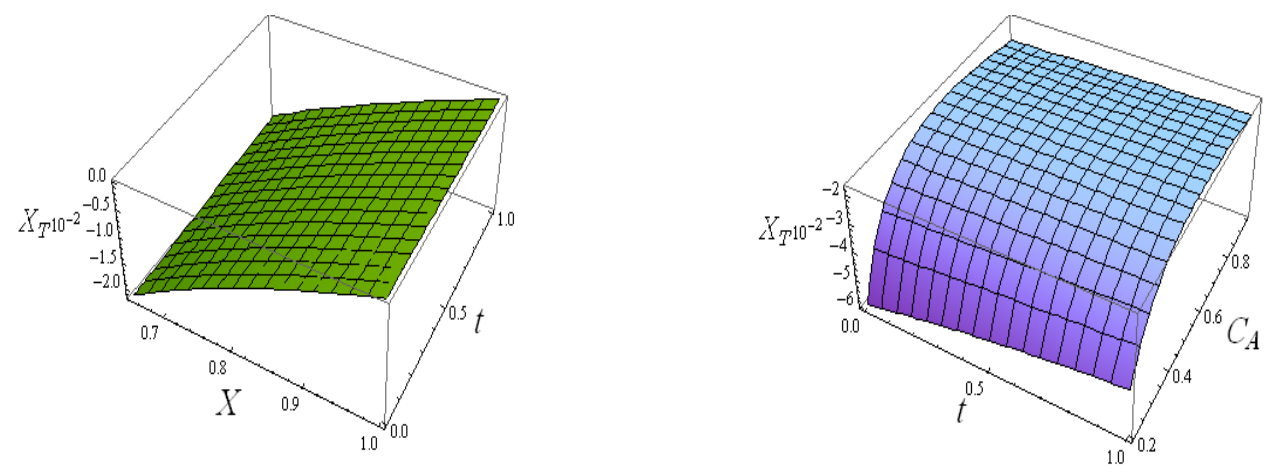

Figure 7. (a) Thermodynamic force $X_{n}$ vs. $t$ and $\chi$ at $C_{A}=0.5, \mathrm{y}=0.3$. (b) Thermodynamic force ${ }_{n}$ vs. $t$ and $C_{A}$ at $\chi=0.66, \mathrm{y}=0.3$.
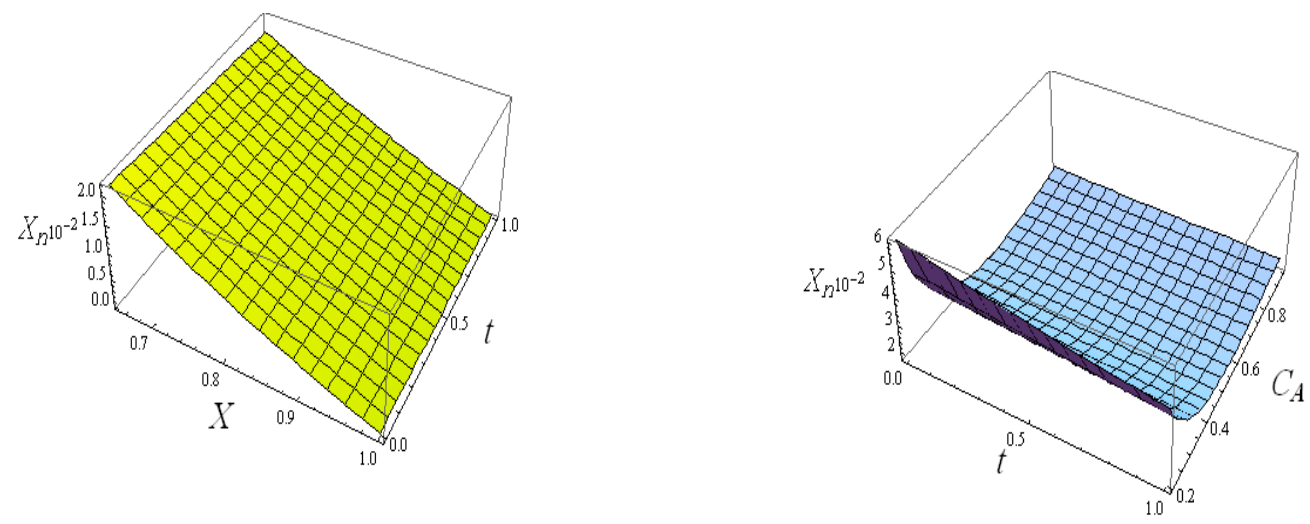

Figure 8. (a) Thermodynamic force $X_{R}$ vs. $t$ and $\chi$ at $C_{A}=0.5, \mathrm{y}=0.3$. (b) Thermodynamic force $X_{R}$ vs. $t$ and $C_{A}$ at $\chi=0.66, \mathrm{y}=0.3$.
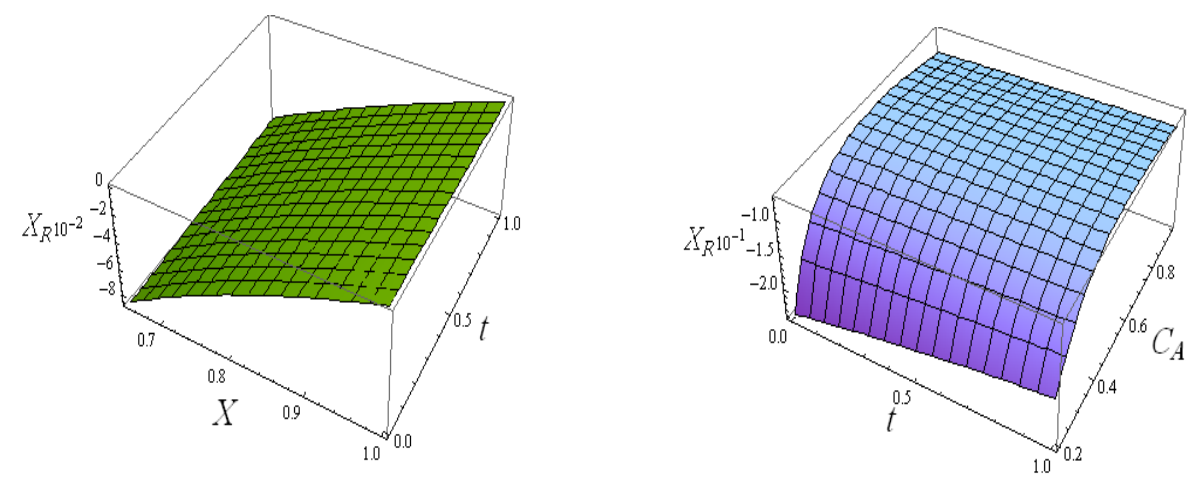

\section{References and Notes}

1. S. Wright," Comparative Analysis of the Entropy of Radiative Heat Transfer and Heat Conduction ", Int. J. of Thermodynamics 2007, 10(1), 27-35. 
2. T. Z. Abdel Wahid " Exact Solution of The Unsteady Krook Kinetic Model and Non-Equilibrium Thermodynamic Study for a Rarefied Gas Affected by a Nonlinear Thermal Radiation Field" Can. J. of Phy., 2013, 91(3): 201-210.

3. F. Sharipov and D. Kalempa, Velocity slip and temperature jump coefficients for gaseous mixtures. III. Diffusion slip coefficient. Phys. Fluids, 2004, 16 (10), 3779-3785.

4. F.J. McCormack, Construction of linearized kinetic models for gaseous mixture and molecular gases, Phys. Fluids, 1973, 16(12), 2095-2105.

5. V.G. Chernyak, V.V. Kalinin, P.E. Suetin, The kinetic phenomena in non-isothermal motion of a binary gas mixture through a plane channel, Int. J. Heat Mass Transfer, 1984, 27 (8), 1189-1196.

6. D. Valougeorgis, Couette flow of a binary gas mixture, Phys. Fluids, 1988, 31 (3), 521-524.

7. A. M. Abourabia and T. Z. Abdel Wahid "Kinetic and Thermodynamic Treatment of a Neutral Binary Gas Mixture Affected by a Nonlinear Thermal Radiation Field." Can. J. of Phys., 2012, 90(2), 137-149.

8. A. M. Abourabia and T. Z. Abdel Wahid "Kinetic and Thermodynamic Treatment for The Rayleigh Flow Problem of an Inhomogeneous Charged Gas Mixture." Journal of Non-Equilibrium Thermodynamics, 2012, 37(1), 1-25.

9. T. Z. Abdel Wahid "Travelling Waves Solution of the Unsteady Flow Problem of a Rarefied NonHomogeneous Charged Gas Bounded by an Oscillating Plate." Mathematical Problems in Engineering J., 2013(ID 503729),1-13.

10. S.R. De Groot, P. Mazur, Non-Equilibrium Thermodynamics, Dover Inc., New York, 1984.

11. C. Cercignani, The Boltzmann Equation and its Application, Springer, New York, 1988.

12. G.A. Bird, "Molecular Gas Dynamics and the Direct Simulation of Gas Flows", Oxford University Press, Oxford, 1994.

13. A. M. Abourabia, T. Z. Abdel Wahid "Solution of The Krook Kinetic Equation Model and NonEquilibrium Thermodynamics of a Rarefied Gas Affected by a Nonlinear Thermal Radiation Field.", Journal of Non-Equilibrium Thermodynamics, 2012, 36 (1), 75-98.

14. A.M. Abourabia and T. Z. Abdel Wahid "The unsteady Boltzmann kinetic Equation and nonequilibrium thermodynamics of an electron gas for the Rayleigh flow problem " Can. J. Phys., 2010, 88 (7), 501-511.

15. T. Z. Abdel Wahid "Kinetic and Thermodynamic Treatment for the Exact Solution of the Unsteady Rayleigh Flow Problem of a Rarefied Homogeneous Charged Gas." Journal of Non-Equilibrium Thermodynamics, 2012, 37(2), 119-141.

16. T. Z. Abdel Wahid, and S. K. Elagan "Kinetic Treatment for the Exact Solution of the Unsteady Rayleigh Flow Problem of a Rarefied Homogeneous Charged Gas Bounded by an Oscillating Plate.", Can. J. Phys. , 2012, 90(10) , 987-998.

17. V. P. Shidloveskiy, "Introduction to Dynamics of Rarefied Gases", Elsevier NY, USA, 1967.

18. S. P. Dawson and A. D. Verga," On The Theory Of Helium Diffusion In Stellar Outer Layers "Rev. Mexicana Astron. Astrof., 1986, 13,.Provided by NASA Astrophysics Data System , 85-100.

19. Doctor of Philosophy Thesis by Mitchell Thomas," Radiation Transfer and Opacity Calculations", California Institute of Technology, Pasadena, California. 1964, Pp 26-27. 
20. D. Mihalas and B.W. Mihalas," Foundation of radiation hydrodynamics " Oxford , NY.,UN. Press, 1984.

21. M. M. Elafify, K. M. Elawadly and Sh.G. Elsharkawy" Prediction of the thermal conductivity of binary gas mixtures using direct simulation Monte Carlo method" Inf. Tech. J., 2003, 3(1), 23-35.

22. J. M. Burt and I. D. Boyd "Evaluation of particle method for the ellipsoidal statistical Bhatnagar Gross Krook equation" the $44^{\text {th }}$ AIAA Aerospace Sciences Meeting and Exhibit, Reno, NV., 2005, AIAA 2006-989.

23. Lees, L. and Liu, C.-Y., "Kinetic Theory Description of Conductive Heat Transfer from A Fine Wire", Physics of Fluids, 1962, 5(10), 1137-1148.

24. Lees, L. "Kinetic Theory Description of Rarified Binary gas mixtures" Journal of The Society for Industrial and Applied Mathematics, 1965, 13(1), 278-311.

25. J.C. Maxwell, L. Rayleigh "Theory of Heat" Longmans, Green, AND Co., London, UK. (1902).

26. F. G. Cheremisin, "Verification of The Nature Of The Approximation Of The Boltzmann Integral By Krook's Kinetic Relaxation Model" Moscow. Translated from Izvestiya Akademii Nauk SSSR, Mekhanika Zhidkosti i Gaza, 1970, 4, 3-7.

27. E.M. Shakhov," Couette Problem for The Generalized Krook Equation Stress-Peak Effect "Izv. AN SSR. Mekhanika Zhidkost I Gaza, 1969, 4,(5), 16-24.

28. Jeans, J. "The Dynamical Theory of Gases." Cambridge University Press, Cambridge, 1904.

29. Gratton, J., Mahajan, S. M. and Minotti, F., "Non-Newtonian Gravity Creeping Flow", International Centre for Theoretical Physics, Trieste, 1988, pp. 1-17,.

30. Nugroho, G., Ali, A. M. S. and Abdul Karim, Z. A., "Towards a new simple analytical formulation of Navier-Stokes equations", World Acd. Sci., Eng. Tec., 2009, 51, 190-194.

31. Clause, P. J. and Mareschal, M., Heat transfer in a gas between parallel plates: Moment method and molecular dynamics, Phys. Rev. A, 1988, 38(8), 4241-4252.

32. Ying-Chu L. W., Flow Generated by a Suddenly Heated Flat Plate, PhD thesis, California Institute of Technology, Pasadena, CA, 1963.

33. V. M. Zhdanov and V. I. Roldugin, "Nonequilibrium Thermodynamics of a Weakly Rarefied Gas Mixture" Zh. Eksp. Teor. Fiz., 1996, 109, 1267-1287.

34. G. Lebon , D. Jou , J. Casas-Vàzquez "Understanding Non-equilibrium Thermodynamics : Foundations, Applications, Frontiers " Springer-Verlag Berlin ,Heidelberg, 2008.

35. F. Sharipov "Reciprocal relations based on the non-stationary Boltzmann equation", Physica A, 2012, 391(5), 1972-1983.

36. R. M. Velasco, L. S. García-Colín and F. J. Uribe " Entropy Production: Its Role in NonEquilibrium Thermodynamics" Entropy Journal, 2011, 13, 82-116; (doi:10.3390/e13010082)

37. A. B. De Castro, "Continuum Thermomechanics" Birkhauser, Verlag, Basel, Switzerland, 2005.

38. G. M. Alves and G. M. Kremer," Classical Kinetic Theory for Binary Gas Mixtures of Monatomic and Polyatomic Gases." J. Non-Equilibrium. Thermodynamic, 1992, 17(2), 171-182.

39. C. F. Delale "The Generalized H Theorem in the Hilbert Space", J. Chem. Phys., 1986, 84 (2), 971 975. (doi:10.1063/1.450545)

40. S. Sieniutycz and R. S. Berry "Canonical formalism, fundamental equation, and generalized thermomechanics for irreversible fluids with heat transfer" Phys. Rev. E , 1993, 47(3), 1765-1783. 
41. T.D. Frank "Pumping and Entropy Production in Non-equilibrium Drift-Diffusion Systems: A Canonical-Dissipative Approach" Eur. J. of Sci. Res., 2010, 46(1), 136-146.

42. L. M. Martyushev, V. D. Seleznev " Maximum entropy production principle in physics, chemistry and biology " Physics Reports, 2006, 426(1), 1 - 45.

43. V.I. Roldughin , V.M. Zhdanov"" Non-equilibrium thermodynamics and kinetic theory of gas mixtures in the presence of interfaces" Advances in Colloid and Interface Science, 2002, 98(2), 121-215.

44. F. Sharipov, Onsager-Casimir reciprocity relations for a mixture of rarefied gases interacting with a laser radiation. J. Stat. Phys., 1995, 78(1/2), 413-430.

45. F. Sharipov, Onsager-Casimir reciprocity relations for open gaseous systems at arbitrary rarefaction. III. Theory and its application for gaseous mixtures. Physica A, 1994, 209(3-4), 457-476.

46. A. M. Abourabia, M. A. Mahmoud, W. S. Abdel Kareem"Unsteady heat transfer of a monatomic gas between two coaxial circular cylinders" Journal of Applied Mathematics, 2002, 2 (3), 141-16.

47. W. W. Liou and Y. Fang, "Microfluid Mechanics principles and Modeling" The McGraw-Hill Companies, Inc, NY, USA, 2006.

48. S. Naris, D. Valougeorgis, D. Kalempa and F. Sharipov "Gaseous mixture flow between two parallel plates in the whole range of the gas rarefaction.", Physica A, 2004, 336(3-4), 294-318.

49. J.D. Huba., NRL plasma formulary, United States. Office of Naval Research, Naval Research Laboratory (U.S.), Washington, D.C., 2011.

(C) 2014 by the authors; licensee MDPI, Basel, Switzerland. This article is an open access article distributed under the terms and conditions of the Creative Commons Attribution license (http://creativecommons.org/licenses/by/3.0/). 